\title{
Radiological findings in carcinoma of the colon complicating chronic ulcerative colitis
}

\author{
JOHN J. FENNESSY, MARSHALL B. SPARBERG, AND JOSEPH B. KIRSNER \\ From the Departments of Radiology and Medicine, The University of Chicago, \\ Chicago, Illinois, U.S.A.
}

There is now general agreement that neoplastic changes occur more frequently in the colon involved with ulcerative colitis than in the undiseased colon. We have reviewed the records of all patients with chronic ulcerative colitis and carcinoma of the colon seen at The University of Chicago hospitals and clinics to determine if there is any characteristic radiological appearance of the colon in those patients who subsequently developed a carcinoma and also to evaluate the gross radiological appearance of the tumours. The clinical and radiological findings also have been correlated.

\section{MATERIALS AND METHODS}

Approximately 1,200 patients suffering from ulcerative colitis have been seen at The University of Chicago hospitals and clinics over the last 30 years. Carcinoma of the colon was detected in $35(2.9 \%)$ cases. Radiographs from the radiological examination of the colon performed within six months before the detection of carcinoma were available for review in 23 patients.

A total of 39 separate primary carcinomas were detected radiologically, clinically, at operation, or at necropsy in the 23 patients for whom complete radiological records were available. For the purpose of this report, nine of these tumours have been excluded from the radiological evaluation for the following reasons: five patients with multiple metachronous tumours had no contrast examinations of the colon performed within six months before the clinical detection of one of these tumours. Two additional neoplasms occurring simultaneously in another patient have also been excluded for the same reason. One recurrent carcinoma in situ is excluded since no contrast examination was performed within six months before the detection of the carcinoma, and a tumour involving the ileocaecal valve was excluded, since only a small bowel examination was performed. In an additional 12 patients, the radiological records are less complete, since no colonic examinations were performed within six months before detection of the tumour. This report is based on the findings in the 23 patients where a full record is available; some information has been included from the remaining 12 patients where this appeared appropriate.
RESULTS

Thirty primary carcinomas potentially detectable by the radiologist in 23 patients were available for review. The radiologist identified $21(70 \%)$ of these lesions and made a positive diagnosis of carcinoma in only $12(40 \%)$. For the remaining nine lesions (five polyps, four 'strictures'), the impression of the radiologist was either 'benign', 'probably benign', or no opinion was given as to whether the lesion was benign or malignant. Carcinoma was diagnosed or suspected clinically in $11(36.7 \%)$ of the tumours. Four tumours $(13 \%)$ overlooked by the radiologist were detected on proctoscopic examination, and one of the other lesions was strongly suspected clinically. The combined clinical and radiological accuracy in detecting or strongly suspecting carcinoma was $57 \%$ (17 of 30). Three of the lesions overlooked by both the clinician and radiologist were present in patients with other simultaneous primary carcinomas of the colon diagnosed both radiologically and clinically. In one patient with an annular carcinoma of the descending colon, a second primary tumour lay several centimetres proximal to the main lesion and was not detected until the bowel was examined by the pathologist. In another patient, two other tumours were present proximal to an obstructing lesion in the descending colon. One or more tumours were overlooked or misdiagnosed by the radiologist in 13 patients.

The tumours identified by the radiologist were typical carcinomas; the most common gross radiological appearance was that of annular constriction (Fig. 1). Several large masses also were seen and there were two instances of total obstruction by a malignant mass (Fig. 2). The lesions potentially diagnosable, but overlooked by the radiologist, are of particular interest. In four patients, areas of localized narrowing were considered to represent benign strictures (Fig. 3), and one area of localized narrowing was overlooked entirely (Fig. 4). A constricting lesion in the sigmoid colon detected 


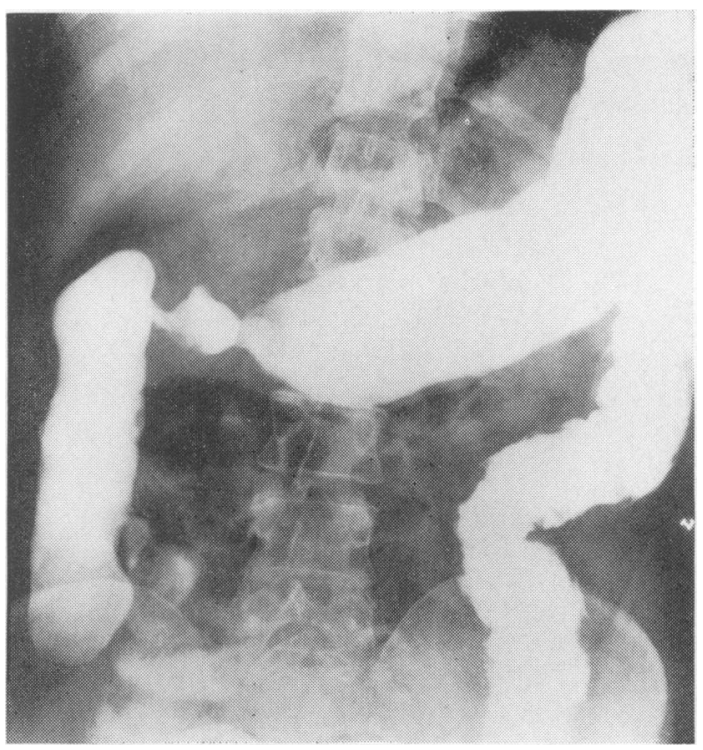

FIG. 1

FIG. 1. This 30-year-old male developed chronic ulcerative colitis at age 19. The symptoms were moderately severe and had been present continuously since the onset. Radiological examination of the colon, performed because of sudden massive haemorrhage, demonstrated a large, ulcerated, annular carcinoma of the hepatic flexure. Moderately severe changes of ulcerative colitis involved the entire colon, together with 'backwash ileitis'; large, deep ulcerations were identified in the descending colon. Laparotomy revealed that the tumour had infiltrated the liver, with multiple mesenteric metastases. The patient died several months later.

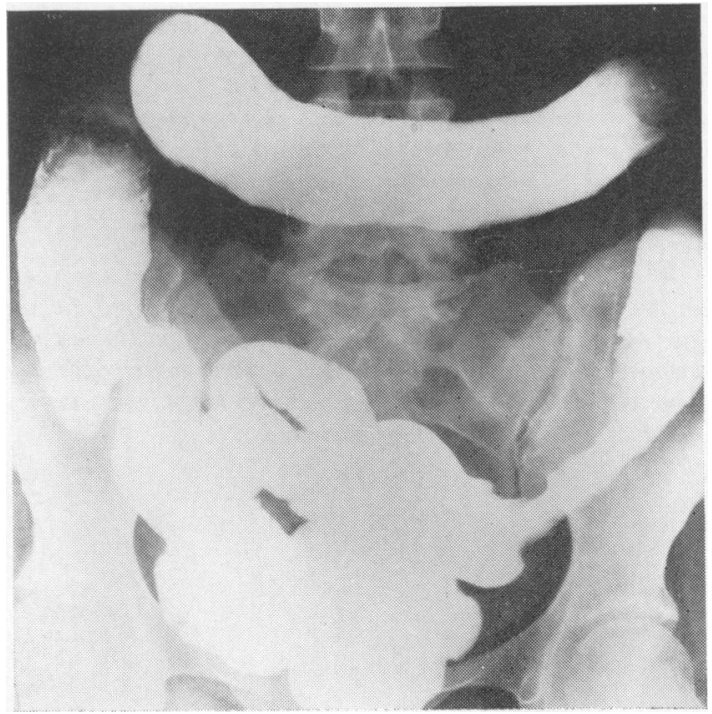

FIG. 3

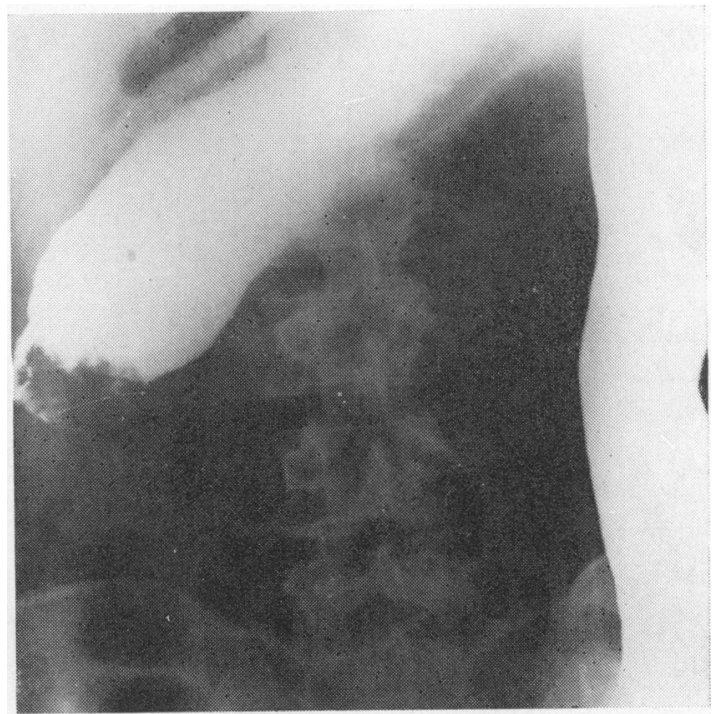

FIG. 2

FIG. 2. This 25-year-old man developed ulcerative colitis at age 20. Mild but continuous symptoms were noted for six years. At this time the patient presented with symptoms consistent with an exacerbation of colitis. Radiological examination of the colon revealed a large fungating carcinoma occluding the colon at the level of the hepatic flexure, with evidence of moderately severe colitis distally.

Operation disclosed a duodeno-colic fistula. A wide resection, including the right side of the colon, the entire fistula, and a wedge of duodenum, was performed. The tumour was found infiltrating the fistula; two of nine lymph nodes contained carcinoma. The patient subsequently remained well for 14 years, when a second carcinoma was detected in the rectum on a routine proctoscopic examination. The remainder of the colon was then removed and the patient has remained well for more than a year.

This case demonstrates a surprisingly good survival following vigorous surgical therapy of the original lesion and also illustrates the ever-present danger of a second colonic neoplasm when the entire colon is not removed.

FIG. 3. E.B., a 49-year-old female, had experienced moderately severe continuous symptoms of ulcerative colitis for 15 years. At the time of this examination, a change in the symptoms was noted, with the onset of pain, fever, and weight loss. Anaemia also was detected. Radiological examination revealed severe changes of ulcerative colitis throughout the colon with narrowing, shortening, and obliteration of the haustra and the mucosa, widening of the ileocaecal valve and 'backwash ileitis'. A narrowing in the distal descending colon was considered to represent an inflammatory stricture. Symptomatic therapy produced temporary relief. However, six months later, a second colon examination again revealed the stricture, now identified as probably a neoplasm.

Operation disclosed an inoperable mucinous adenocarcinoma. A colostomy was performed and the patient died within a year. While the gross appearance of this tumour is unusual, the eccentricity of the lumen proximally and the overhanging margins distally are typical of carcinoma. 


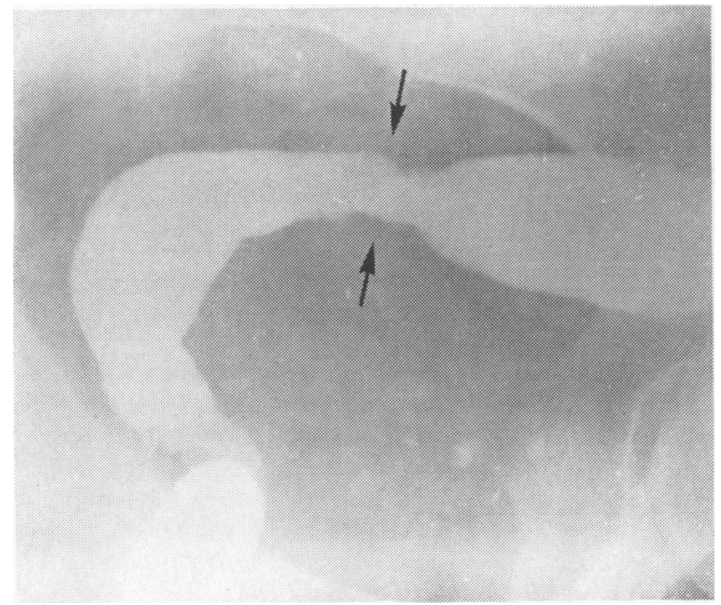

FIG. 4

FIG. 4. This 31-year-old male developed moderately severe colitis with intermittent symptoms for 12 years. Rectal polyps previously had been fulgurated at another hospital; one of these polyps represented 'grade 1 carcinoma'.

The patient had been asymptomatic for five years before the detection of a rectal carcinoma on routine proctoscopic examination. Radiological examination of the colon revealed mild changes of colitis distal to the ascending colon, with severe changes in the rectum, including narrowing and loss of Houston's folds. A localized concentric and irregular narrowing of the rectosigmoid colon representing the carcinoma was overlooked by the radiologists.

A local resection of the tumour was performed. One year later proctoscopic examination revealed recurrent tumour at the anastomosis. Subsequently, the entire colon was removed. The patient later died from metastatic carcinoma. This case again illustrates that carcinoma may arise in the colon years after the symptoms of colitis have subsided.

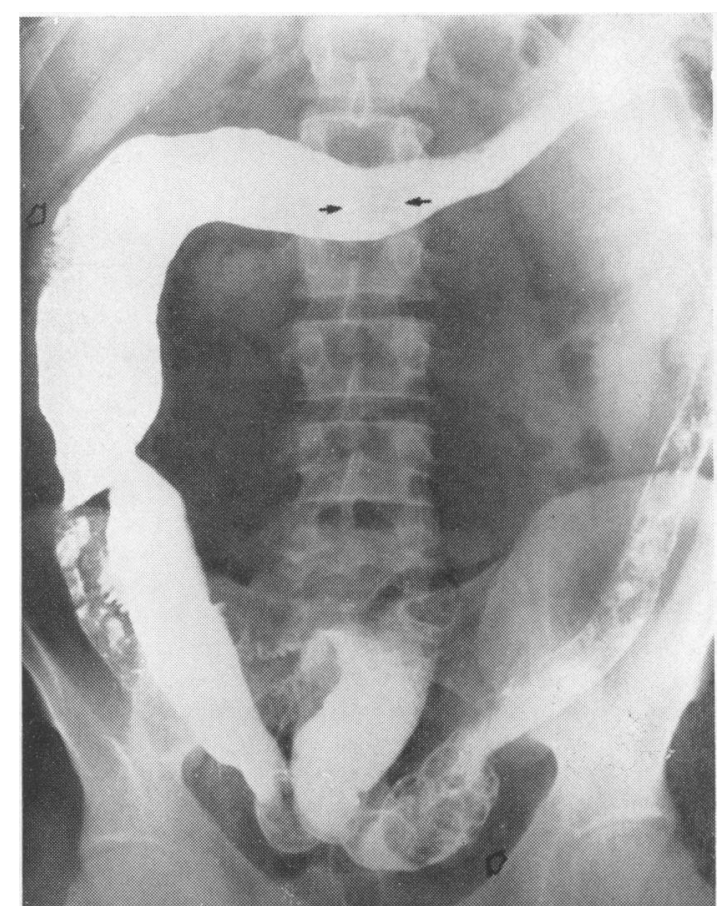

FIG. 5

FIG. 5. B.M., a 29-year-old male, developed ulcerative colitis at age 22. Symptoms were moderately severe and had been present intermittently. On this occasion, a change in symptoms included abdominal pain and vomiting. Radiological examination of the colon revealed severe ulcerative colitis throughout, with concurrent disease of a long segment of the terminal ileum. A large polypoid mass was present in the sigmoid colon, with similar but smaller masses in the transverse and ascending colon. Small polyps were identified elsewhere in the colon.

A subtotal colectomy with ileo-proctostomy was performed and six polypoid masses were detected in the specimen. Three of these masses represented 'low-grade' adenocarcinoma. Six years later, another carcinoma was detected in the rectum. The patient refused further surgery and died four years later of carcinoma. 


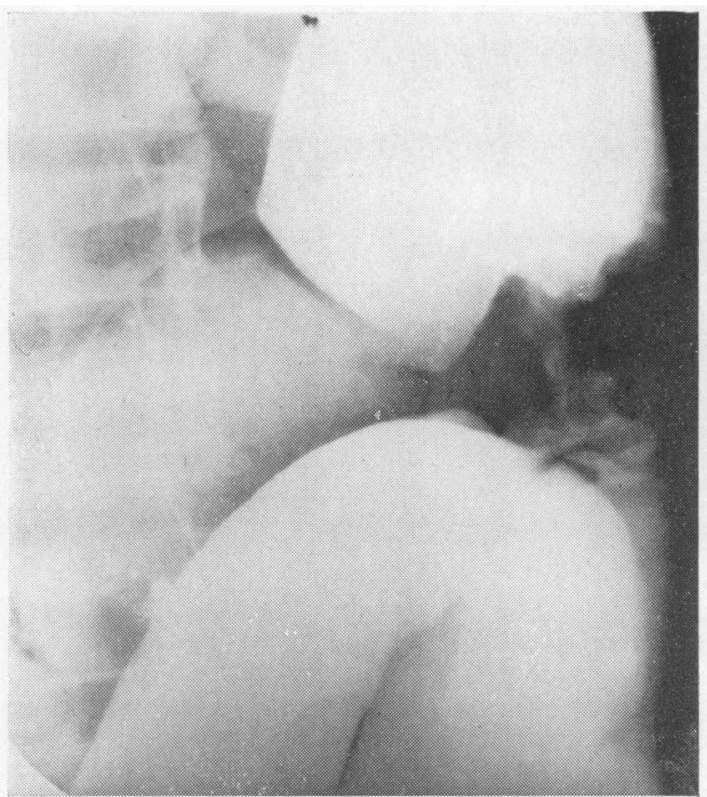

FIG. $6 \mathrm{a}$

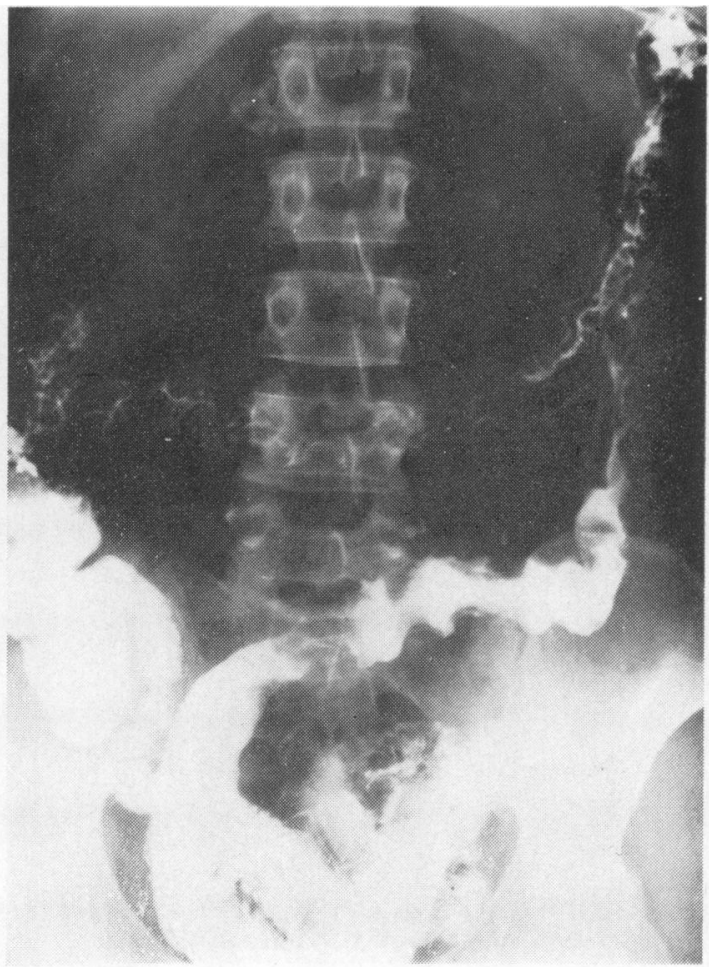

FIG. $6 c$

FIG. 6. R.G., a negro female, developed intermittent bloody diarrhoea at age 17. Ulcerative colitis was diagnosed at age 37 and a colonic examination revealed mild ulcerative colitis distal to the midtransverse colon. One year later, anaemia was found. Exfoliative cytology was normal and a colonic examination showed no change. Four months later the patient experienced a severe colonic haemorrhage and at this time the examination revealed an annular carcinoma just distal to the splenic flexure (a view). The filled views of the colon reveal a grossly normal colon (b view). The postevacuation films (c view), however, show progressive mucosal thickening and obliteration from the transverse colon distally.

Of the colon $28 \mathrm{~cm}$ was resected and a second, small and unsuspected tumour was found several centimetres proximal to the annular carcinoma.

For two years subsequently the patient remained well. Occult blood was then detected in the stool. Radiological examination of the colon on two occasions revealed only minor evidence of colitis. Exfoliative cytology of the colon, however, disclosed cancer cells. Because of this finding, the entire remaining colon was removed and a $12 \mathrm{~mm}$ plaque of cancer was found in the specimen. The patient died of carcinoma four months later.

This case illustrates the value of colonic cytology in cases where the radiological examination reveals no significant abnormalities, but there is a strong clinical suspicion of carcinoma. 


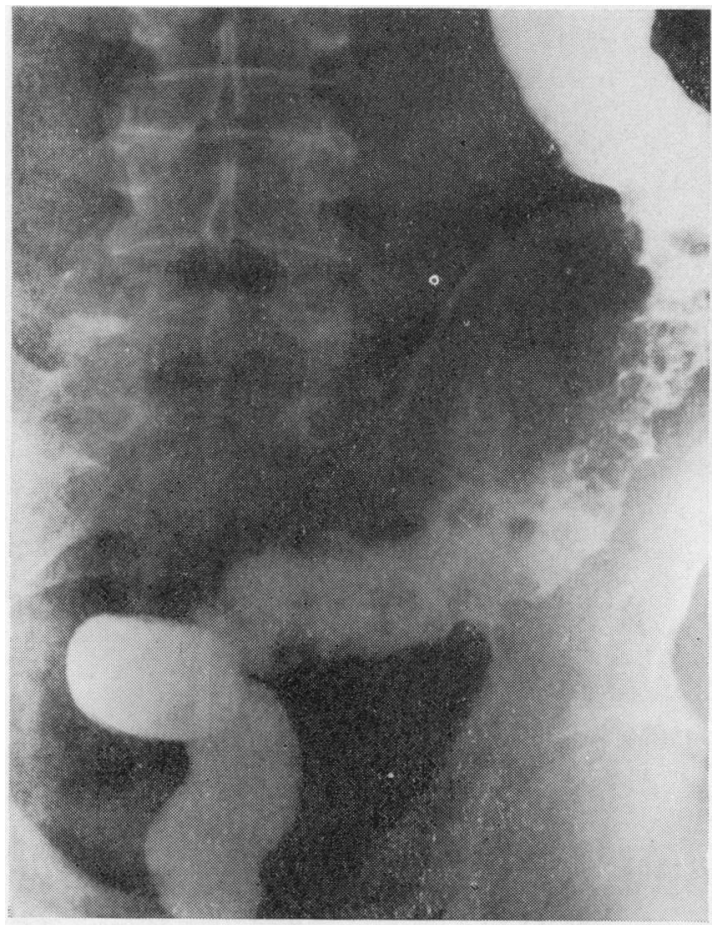

FIG. 7a

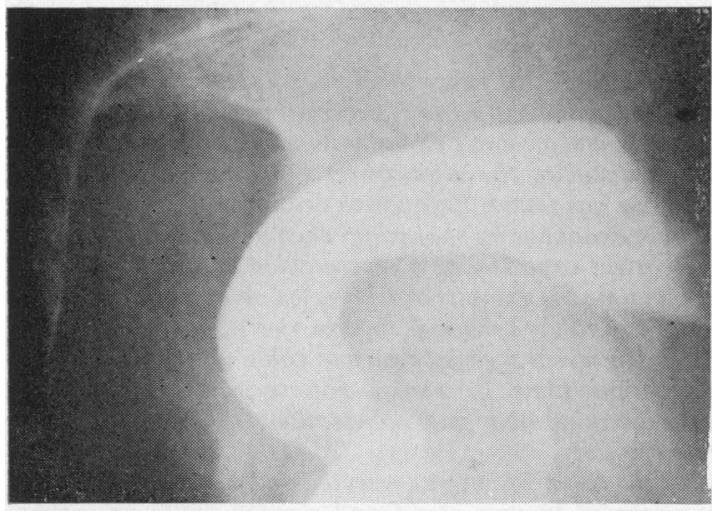

FIG. $7 b$

FIG. 7. This 54-year-old male patient developed moderately severe colitis with intermittent symptoms at age 33. Pseudopolyps had been identified radiologically in the sigmoid and descending colon in the past and these lesions had increased in size and number over a period of several years (a view). The colon was normal proximal to the splenic flexure. The rectum, however, was severely deformed with obliteration of the folds, narrowing of the lumen and pronounced thickening of the presacral space (b view). Proctoscopic and radiographic examinations were performed because of a change in symptoms, and both

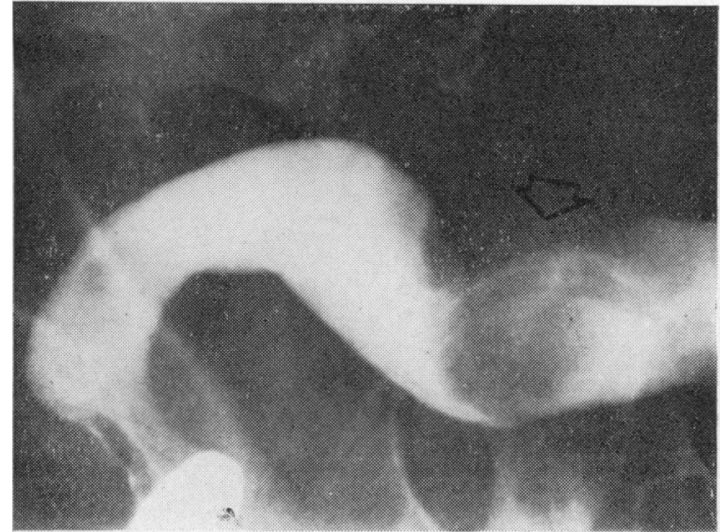

FIG. 7c

procedures revealed a large carcinoma of the sigmoid colon, distal to the area involved by pseudopolyposis (c view.).

A partial colectomy was performed, revealing a mucusproducing adenocarcinoma in the distal sigmoid colon. Metastases were found in 10 of 18 lymph nodes. The patient died two years later.

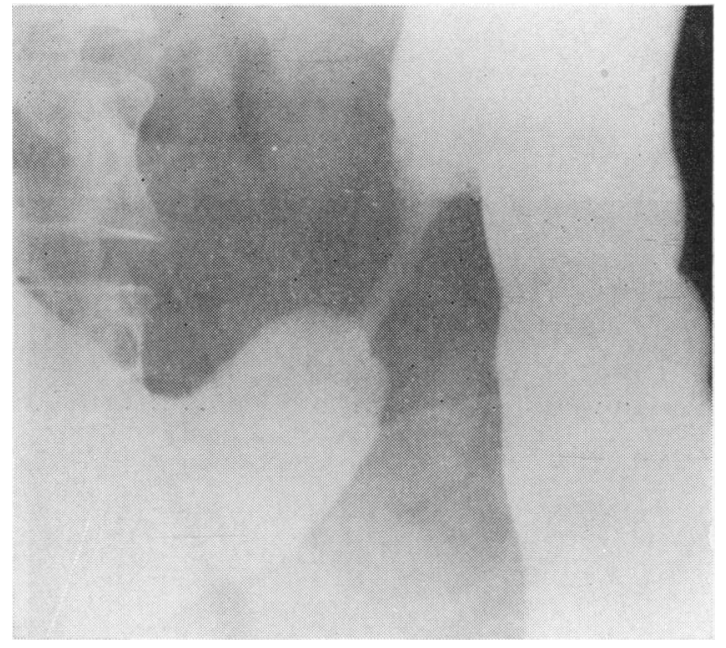

FIG. 8

FIG. 8. This 36-year-old male patient had experienced continuous, moderately severe symptoms of ulcerative colitis for 20 years. Because of a change in symptoms, with the onset of cramping abdominal pain, carcinoma was suspected and a colonic examination was performed. A stenosis was identified in the distal transverse colon. This lesion had features of both a benign and malignant stricture. However, the presence of overhanging margins proximally was felt to be indicative of tumour, an impression confirmed at laparotomy when a partial colectomy was performed. The patient died six years later of hepatic metastases. 
proctoscopically was missed by the radiologist because of failure to obtain spot films of the region. A carcinoma of the appendix involving the caecum was also overlooked. Several polypoid lesions were evaluated as benign, or no impression was recorded as to benignancy or malignancy. Several small tumours were also overlooked; even in retrospect and knowing the precise site of the lesion as determined at operation or on proctoscopic examination, these cannot be detected. An air-contrast examination was performed in only one of the patients in whom two annular simultaneous carcinomas were present, but this added no helpful information. Multiple synchronous carcinomas are common in these patients but in only one instance was the second primary tumour detected by the radiologist.

The most striking radiological finding in both the group for whom the radiological records are complete and among those with radiological records less complete is the extent of the ulcerative colitis. In the vast majority $(82 \%)$ the disease affected the entire colon, and in $44 \%$ it also involved the terminal ileum for a variable distance (Fig. 5). The colitis was less extensive in the remainder but in no case was the disease limited to the sigmoid colon or rectum. While the colitis usually was extensive, the changes were not necessarily severe. Severe disease with narrowing, shortening, and obliteration of the haustra was present in $36 \%$. Moderately severe changes were present in $41 \%$, and relatively mild changes were identified in the remainder (Fig. 6). There was a close correlation between the clinical and the radiological evaluation of the severity of the disease. A finding of particular interest was that, even though the changes in the colon might be consistent with moderately severe or mild colitis, the changes in the rectum almost invariably were severe. Narrowing of the ampulla of the rectum with obliteration of Houston's folds was the usual finding and, in those patients in whom lateral films of the rectum were available, thickening of the presacral space also was present almost invariably (Fig. 7). These changes, however, are an index of the chronicity of the disease as we have noted previously (Fennessy, Sparberg, and Kirsner, 1966). The changes of ulcerative colitis usually were static or progressive. However, occasionally the appearance of the colon had improved significantly at the time of detection of the carcinoma.

Pseudopolyposis was uncommon. Pseudopolyps were identified in $17 \%$ but were not associated anatomically with the development of carcinoma. In one patient, a tumour arose immediately distal to an area of severe pseudopolyposis, but this lesion was definitely separate from the polyps (Fig. 7). One or more 'synchronous' carcinomas were present in almost one-fourth of the patients $(23.8 \%)$. Three of these synchronous carcinomas arose in one patient and each was thought to represent a benign polyp. The presence of the second lesion or of multiple tumours was recognized in only one patient.

Radiological evidence of active colitis at the time of detection of a carcinoma was uncommon and was apparent in only four patients (Fig. 1). Skeletal metastasis from carcinoma of the colon is uncommon (Weston and Feit, 1966) and was detected in only one patient; this was a destructive lesion involving the lower lumbar spine and may have represented local extension of the tumour rather than haematogenous spread.

\section{CLINICAL FINDINGS}

The diagnosis of carcinoma was rarely made before radiological or proctoscopic examination. In some cases, however, carcinoma was strongly suspected because of the onset of pain or obstructive symptoms. Occasionally, a diagnosis of carcinoma was made or confirmed by colon cytology (Goldgraber, Humphreys, Kirsner, and Palmer, 1958; Goldgraber and Kirsner, 1964). The patients usually presented with an exacerbation or recurrence of the symptoms of ulcerative colitis. The average age of the patients at the time of onset of colitis was 24.2 years $(2.5$ to 68 years). The average age at the time of detection of the carcinoma was $37 \cdot 7$ years. The average duration of colitis before the detection of carcinoma was 16.9 years (six months to 38 years) and the average at the time of death from carcinoma was 40.6 years $(16.5$ to 72 years).

In most cases, the patients experienced moderately severe symptoms of colitis $(56 \%)$. Symptoms were severe in $33 \%$ and mild in the remainder. Symptoms of colitis tended to be intermittent rather than continuous $(53.5 \%)$.

Of the entire group of 35 patients, 23 developed one carcinoma only. Fifteen of these 23 patients with single tumours have died of carcinoma. Twelve patients $(34 \%)$ developed two or more carcinomas; of these, eight patients developed two or more synchronous carcinomas. Eight of the patients with multiple tumours have died of carcinoma, and two have been lost to follow-up. One patient remains alive eight years following the removal of two synchronous tumours; the other survivor had a single carcinoma of the ascending colon resected and 14 years later developed a second primary in the rectum. He remains alive one year after colectomy.

The mean postoperative survival following curative operative procedures (excluding postoperative deaths) was 3.9 years. Nine patients were treated 
originally by a partial colectomy or local resection of the tumour, later followed by a total colectomy at intervals from one month to 14 years (mean $7 \cdot 8$ years). It is of some interest that six $(67 \%)$ had developed another carcinoma at the time of the colectomy. In three of these patients, the new carcinoma was unsuspected. Seven $(37 \%)$ of the 19 patients initially treated by local resection of the tumour or by partial colectomy later developed another primary lesion in the remaining colon. One patient had a recurrence of tumour at the anastomosis.

Three patients were treated originally for polypoid adenoma malignum by polypectomy. One of these patients died 13 years later from a carcinoma arising in the region from which the polyp was removed.

A total of 57 primary carcinomas arose in the entire group of 35 patients. Forty-five of these tumours were proximal to the rectosigmoid junction $(78 \%)$; nine were present in the sigmoid colon; 13 in the descending colon; seven in the transverse colon; four in the hepatic flexure; six in the ascending colon; and six involving the caecum, appendix, or terminal ileum. Of the group of 30 tumours arising in 23 patients, which forms the basis of this report, 26 of the tumours were proximal to the rectosigmoid junction $(87 \%)$.

\section{DISCUSSION}

The increased risk of carcinoma arising in the colon involved with ulcerative colitis is well established. The exact incidence of malignant change varies, usually ranging from $1 \%$ to $14 \%$ (Edwards and Truelove, 1964), whereas in the population at large the incidence is $0.06 \%$ (Weckesser and Chinn, 1953). It is also accepted that the likelihood of developing a carcinoma increases with the duration of the colitis (Lagercrantz, 1955; Rosenqvist, Lagercrantz, Öhrling, and Edling, 1959; Slaney and Brooke, 1959), reaching definite clinical significance after 10 years (Hinton, 1966) and rising to an incidence of $56 \%$ after 30 years (de Dombal, Watts, Watkinson, and Goligher, 1966). These patients develop carcinoma at an unusually early age (Shands, Dockerty, and Bargen, 1952; Rosenqvist et al, 1959; Slaney and Brooke, 1959; Hodgson and Sauer, 1961; Edwards and Truelove, 1964). The mean age of the time of detection of carcinoma in our patients was 37.7 years, in striking contrast to those individuals with an intact colon who develop carcinoma at an average age of 57.8 years (Swinton and Counts, 1956).

In the group of patients reported here, the mean interval from onset of colitis to detection of carcinoma was 16.9 years. This is remarkably similar to the duration of colitis reported from other institu- tions, both in the United States and abroad (Shands et al, 1952; Rosenqvist et al, 1959; Hodgson and Sauer, 1961; Diaz, Farmer, and Brown, 1965). Welch (Welch and Hedberg, 1965), however, noted an average duration of nine years before the detection of carcinoma, and in Shands' series(Shandset al, 1952) $24.7 \%$ had ulcerative colitis for less than 10 years.

It also has been noted that carcinoma is particularly liable to arise in the colon with extensive involvement (Edwards and Truelove, 1964; MacDougall, 1964) and is less likely or does not occur any more frequently than in the intact colon when the changes are limited to the distal segment of the colon (MacDougall, 1964). While extensive involvement was the rule in our patients, the majority had moderate or mild stigmata of colitis rather than more severe changes. MacDougall has stated that if the inflammatory changes are limited to the distal colon (MacDougall, 1964) or if the colon is radiologically normal (MacDougall, 1964), there is no increased risk of carcinoma. However, Edling, Lagercrantz, and Rosenqvist (1959) described two cases where the colon was radiologically normal at the time the carcinoma was detected. Severe changes were commonly present in the rectum, with narrowing of the lumen, obliteration of the folds, and thickening of the presacral space, but these alterations also may be seen in ulcerative proctitis and proctocolitis (Fennessy, Sparberg, and Kirsner, 1966), where there is no increased risk of carcinoma (Sparberg, Fennessy, and Kirsner, 1966).

A particularly discouraging aspect of the problem is the very low rate of diagnosis of the tumours by both the radiologist and clinician. This is in marked contrast to the results in the carcinomas arising in the intact colon where the radiologist will establish a diagnosis in $95 \%$ of the lesions proximal to the reach of the proctoscopist (Lauer, Carlson, and Wollaeger, 1965). We do not agree with Hodgson and Sauer (1961) that the radiologist should not concern himself with that portion of the colon within the reach of the proctoscopist. While we agree that the radiologist is less likely to detect lesions in the rectum, this does not eliminate the necessity of examining that region, since on occasion lesions may be noted that have been overlooked by the proctoscopist (Osborne, Pattinson, and Ward, 1956). Difficulty in establishing a diagnosis of carcinoma by radiological methods has been noted by others (Counsell and Dukes, 1952; Slaney and Brooke, 1959). Rosenqvist et al (1959), however, have reported that the fully developed carcinoma is easily diagnosed, and Edling et al (1959) have stated that in all cases the tumours are well demar- 
cated. Brooke (1961), on the contrary, attributes the poor prognosis in these patients to the difficulty in detecting the tumours. In some cases, the inability of the radiologist to detect the lesion is easily appreciated, since the tumours may be extremely small and cannot be seen on the films even in retrospect. Hultborn (1952) has stated that the smallest lesion potentially detectable radiologically is $1.0 \mathrm{~cm}$ or slightly less, and Hinton (1966) feels that air contrast is essential to detect these small lesions. Boijsen and Reuter (1966) have been able to detect colonic carcinomas as small as $1.5 \times 1.0 \mathrm{~cm}$ in a patient suffering from ulcerative colitis by means of mesenteric angiography. This technique may prove useful in the detection of carcinoma in selected cases.

Many of the tumours overlooked or misdiagnosed by the radiologist represented 'strictures'. Hinton (1966) has stated that the presence of a stricture in patients with ulcerative colitis should be regarded as suggesting carcinoma. We believe that a stricture in a patient with inflammatory disease of the large bowel represents either a carcinoma or the disease process is probably granulomatous colitis. If the diagnosis of ulcerative colitis is confirmed, the stricture should be considered to represent a tumour until the contrary has been proved. There is some disagreement in the literature as to the frequency of benign inflammatory stricture in chronic ulcerative colitis. Marshak, Bloch, and Wolf (1963) feel that these lesions are rare, whereas de Dombal et al (1966) have identified strictures in $11.2 \%$ of their patients and in no case detected a carcinoma at a stricture. Our experience is in accord with that of Marshak et al, ie, that true strictures are uncommon in ulcerative colitis. The chief importance of the stricture is its differentiation from a neoplasm. While this differentiation may be impossible in some cases, in other instances, including the majority of the lesions overlooked in this series, definite differences between the benign and malignant lesions can be noted (Fig. 8). All of the elongated malignant strictures in this group had clearly demarcated margins. The lumen in the area of the stricture was irregular and eccentric, and occasionally localized masses intruded upon the lumen at the point of stricture. Each of the lesions overlooked in this series undoubtedly would have been diagnosed as carcinoma if it were present in a colon uninvolved with ulcerative colitis. The difficulty in establishing a radiological diagnosis of tumours in these cases is particularly frustrating since, unlike the carcinoma arising in the intact colon where $60-70 \%$ of the lesions may be diagnosed proctoscopically (Hultborn, 1952), in patients with colitis the majority of the lesions lie proximal to the sigmoid. Of the 57 carcinomas arising in all patients included in this series, 45 or $79 \%$ were proximal to the rectosigmoid junction. Edling and Eklöf (1961) have noted a similar distribution of the tumours in their series and state that a physician relying only on proctosigmoidoscopy will overlook the majority of tumours occurring in patients with ulcerative colitis. Similar findings have been noted by others (Hinton, 1966). The symptoms in these patients are frequently confusing, the majority presenting with symptoms of recurrence or exacerbation of chronic ulcerative colitis (Hinton, 1966; Slaney and Brooke, 1959). MacDougall (1964) has stated that it is unsafe to suppose that symptoms may be helpful in the early diagnosis of carcinoma. The majority of our patients experienced moderately severe symptoms of colitis and the intermittent form of the disease was slightly more common than the continuous pattern. Lagercrantz (1955) noted intermittent symptoms of colitis in $78.8 \%$ of his patients, while Edwards and Truelove (1964) noted the highest incidence of carcinoma in those individuals with chronic continuous ulcerative colitis.

The multiplicity of the tumours arising in these patients also is noteworthy (Goldgraber, Humphreys, Kirsner, and Palmer, 1958; Hodgson and Sauer, 1961; Goldgraber and Kirsner, 1964; Diaz et al, 1965; Fennessy et al, 1966; Hinton, 1966). Two or more primary carcinomas arose in $34 \%$. Moertel, Bargen, and Dockerty (1958) detected multiple carcinomas in $4.6 \%$ of a large series of patients developing carcinoma with an intact colon. Hojman, Garriz, and Markman (1966) detected a somewhat lower incidence of meltiple primary tumours in the intact colon $(2.02 \%)$, but they noted that if in one colon cancer develops the risk of developing a second lesion is 11 times greater than in the population at large. Synchronous primary carcinomas arose in $23 \%$ of our group of patients, whereas Moertel et al (1958) detected synchronous carcinomas in only $2.6 \%$ of patients without ulcerative colitis. This tendency to multiple colonic neoplasia increases the diagnostic difficulty, as was demonstrated by our series where in only one patient was the second synchronous tumour detected before operation. The likelihood of developing a new primary carcinoma following a partial resection of bowel, $37 \%$ in our series, also has been noted by others (Farmer and Brown, 1964; MacDougall, 1964; Slaney and Brooke, 1959). This experience warrants reaffirmation of the need for a total colectomy once a carcinoma has been detected in the colon of these patients (Goldgraber and Kirsner, 1964; Shands et al 1952). Rosenqvist et al (1959), however, noted 
only two of 26 carcinomas in the rectum and feel justified in preserving that portion of the bowel.

While Svartz (Svartz and Gillnäs, 1958; Svartz and Ernberg, 1949) has emphasized either a direct relationship between pseudopolyps and carcinoma or the predisposition of pseudopolyps to carcinoma, this relationship has not been our experience. Edwards and Truelove (1964) and others (Kanin, 1965) have noted no relationship between pseudopolyps and carcinoma other than that they are a measure of severe disease and may be seen in patients especially likely to develop carcinoma. While Shands et al (1952) have described a high incidence of pseudopolyps in patients developing multiple carcinomas, we noted pseudopolyps in association with multiple carcinomas in only two patients.

The survival of these patients is usually poor (Edling and Eklöf, 1961). In spite of this generally poor prognosis, occasional patients survive for long periods, despite the finding of lymph node metastases at the time of surgery (Goldgraber and Kirsner, 1964) (Fig. 2). Whether the poor outlook is a result of a different tumour growth pattern from carcinoma arising in the colon unaffected by ulcerative colitis or is related to the early age at which the tumours arise or to the difficulty in detecting these lesions at an early stage has not been determined. Perhaps all of these factors contribute to the prognosis. Brooke (1961) has related the poor prognosis to the difficulty in early diagnosis rather than to inherent virulence, and Coffey and Cardenas (1964) have noted that carcinomas of the colon in young adults are uniquely fulminant with a poor prognosis.

In the intact colon uninvolved with ulcerative colitis, carcinomas are more common in male patients, with a ratio of 2 to 1 . The preponderance of male patients with colon carcinoma and ulcerative colitis has been noted in other series, such as those of Shands et al (1952), Rosenqvist et al (1959), and Diaz et al (1965). Weber (1965), however, has stated that the lesions are more common in females. In our series, the ratio was approximately 1 to 1 , with 18 tumours arising in male patients and 17 in females. This proportion perhaps is unusual since, as has been pointed out by Kirsner (1958), ulcerative colitis is somewhat commoner in females than in males.

There is some disagreement in the literature as to whether or not routine radiological examinations of the colon at intervals is indicated in all patients with chronic ulcerative colitis. Rosenqvist et al (1959) have stressed the importance of radiological followup, and Farmer and Brown (1964) have stated that periodic evaluation of all patients with chronic ulcerative colitis is mandatory. Svartz and Ernberg (1949) hesitate to perform annual colon examinations because of the increased anxiety of the patients, and Slaney and Brooke (1959) feel that conventional follow-up, even with barium enema, is ineffective, since the carcinoma usually is too far advanced for cure at the time of diagnosis. Dukes' (Dukes, 1964) statement on this problem is worth quoting in this context: 'As soon as possible, the patient should be encouraged to forget that he ever had ulcerative colitis, but his doctor, on the other hand, had better not forget too soon.' He also has stated that these patients require special supervision and regular re-examination. Radiological examination of the colon is not performed at routine intervals on all patients seen at The University of Chicago hospitals and clinics. Usually repeat examinations are performed at approximately two-year intervals, or more frequently where there is a special indication, such as recurrent symptoms. However, since carcinoma of the colon may develop following a long period of quiescence of the colitis (Svartz and Ernberg, 1949; Rosenqvist et al, 1959), any patient presenting with recurrent symptoms after a long remission must undergo prompt radiological examination of the colon.

\section{SUMMARY}

The radiological and clinical findings in a series of patients suffering from chronic ulcerative colitis, complicated by the development of carcinoma of the colon, are reviewed.

Patients with ulcerative colitis develop carcinoma at an unusually early age. The majority of the tumours are situated proximal to the reach of the proctoscopist.

Most of the patients die of the colonic carcinoma.

Multiple synchronous or metachronous tumours of the colon are common in patients with ulcerative colitis. Patients originally treated for colonic carcinoma by a partial bowel resection often develop another tumour in the remaining colon.

Many of the potentially detectable tumours are not recognized clinically or radiologically.

Benign inflammatory stricture of the colon is uncommon in patients with ulcerative colitis. Strictures developing in the colon of these patients must be considered to represent carcinoma until the contrary is proved.

\section{REFERENCES}

Boijsen, E., and Reuter, S. R. (1966). Mesenteric angiography in the evaluation of inflammatory and neoplastic disease of the intestine. Radiology, 87, 1028-1036.

Brooke, B. N. (1961). Malignant change in ulcerative colitis. Dis. Colon Rect., 4, 393-398. 
Coffey, R. J., and Cardenas, F. (1964). Cancer of the bowel in the young adult. Ibid., 7, 491-492.

Counsell, P. B., and Dukes, C. E. (1952). The association of chronic ulcerative colitis and carcinoma of the rectum and colon. Brit. J. Surg., 39, 485-495.

de Dombal, F. T., Watts, J. McK., Watkinson, G., and Goligher, J. C. (1966). Local complications of ulcerative colitis: stricture, pseudopolyposis and carcinoma of the colon and rectum. Brit. med. J., 1, 1442-1447.

Diaz, R. J., Farmer, R. G., and Brown, C. H. (1965). Carcinoma of the colon and ulcerative colitis. Amer. J. dig. Dis., 10, 643-656.

Dukes, C. E. (1964). The control of precancerous conditions of the colon and rectum. Canad. med. Ass. J., 90, 630-635.

Edling, N. P. G., and Eklöf, O. (1961). Distribution of malignancy in ulcerative colitis. Gastroenterology, 41, 465-466.

- - (1961). Radiologic findings and prognosis in ulcerative colitis. Acta chir. scand., 121, 299-308.

- Lagercrantz, R., and Rosenqvist, H. (1959). Roentgenologic findings in ulcerative colitis with malignant degeneration. Acta radiol. (Stockh.), 52, 123-128.

Edwards, F. C., and Truelove, S. C. (1964). The course and prognosis of ulcerative colitis: Part IV. Carcinoma of the colon. Gut, 5, $15 \cdot 22$.

Farmer, R. G., and Brown, C. H. (1964). Colonic carcinoma and ulcerative colitis: multifocal carcinoma occurring after long remission of colitis. Arch. intern. Med., 113, 153-157.

Fennessy, J. J., Sparberg, M., and Kirsner, J. B. (1966). Early roentgen manifestations of mild ulcerative colitis and proctitis. Radiology, 87, 848-858.

Goldgraber, M. B., Humphreys, E. M., Kirsner, J. B., and Palmer, W. L. (1958). Cancer and ulcerative colitis: a clinical-pathologic study. Gastroenterology, 34, 809-846.

- , and Kirsner, J. B. (1964). Carcinoma of colon complicating ulcerative colitis. Dis. Colon Rect., 7, 336-344.

- - (1964). Carcinoma of the colon in ulcerative colitis. Cancer (Philad.), 17, 657-665.

Hinton, J. M. (1966). Risk of malignant change in ulcerative colitis. Gut, 7, 427-432.

Hodgson, J. R., and Sauer, W. G. (1961). The roentgenologic features of carcinoma in chronic ulcerative colitis. Amer.J. Roentgenol., 86, 91-96.

Hojman, D., Garriz, R. A., and Markman, I. (1966). Multiple malignant tumors of the colon and rectum. Dis. Colon Rect. 9, 121-126.

Hultborn, K. A. (1952). Cancer of the colon and rectum. Acta chir. scand. suppl. 172.
Kanin, H. J. (1965). Pseudopolyposis and the cancer problem in ulcerative colitis. Amer. J. Gastroenterol., 43, 484-488.

Kirsner, J. B. (1958). Ulcerative colitis: a challenge. Arch. intern. Med., 101, 3-8.

Lagercrantz, R. (1955). Follow-up investigation of children with ulcerative colitis. Acta paediat. (Uppsala), 44, 302-317.

Lauer, J. D., Carlson, M. C., and Wollaeger, E. E. (1965). Accuracy of roentgenologic examination in detecting carcinoma of the colon. Dis. Colon Rect., 8, 190-197.

MacDougall, I. P. M. (1964). Clinical identification of those cases of ulcerative colitis most likely to develop cancer of the bowel. Ibid., 7, 447-450.

(1964). The cancer risk in ulcerative colitis. Lancet, 2, 655-658.

Marshak, R. H., Bloch, C., and Wolf, B. S. (1963). The roentgen findings in strictures of the colon associated with ulcerative and granulomatous colitis. Amer. J. Roentgenol., 90, 709-716.

Moertel, C. G., Bargen, J. A., and Dockerty, M. B. (1958). Multiple carcinomas of the large intestine. Gastroenterology, 34, 85-98.

Osborne, G., Pattinson, J. N., and Ward, M. W. P. (1956). The value of the lateral view of the rectosigmoid. J. Fac. Radiol. (Lond.), 7, 286-290.

Rosenqvist, H., Öhrling, E., Lagercrantz, R., and Edling, N. (1959). Ulcerative colitis and carcinoma coli. Lancet, 1, 906-908.

Shands, W. C., Dockerty, M. B., and Bargen, J. A. (1952). Adenocarcinoma of the large intestine associated with chronic ulcerative colitis: clinical and pathologic features of 73 cases. Surg. Gynec. Obstet., 94, 302-310.

Slaney, G., and Brooke, B. N. (1959). Carcinoma in ulcerative colitis. Lancet, 2, 694-698.

Sparberg, M., Fennessy, J., and Kirsner, J. B. (1966). Ulcerative proctitis and mild ulcerative colitis. Medicine (Baltimore), 45, 391-412.

Svartz, N., and Gillnäs, T. (1958). In which phase of ulcerative colitis does colonic cancer occur? Amer. J. dig. Dis., 3, 537-548.

- , and Ernberg, T. (1949). Cancer coli in cases of colitis ulcerosa. Acta med. scand., 135, 444-447.

Swinton, N. W., and Counts, R. L. (1956). Carcinoma of the colon and rectum. J. Amer. med. Ass., 161, 1139-1142.

Weber, A. O. (1965). Carcinoma of the colon complicating ulcerative colitis with five-year survival. Calif. Med., 102, 139-141.

Welch, C. E., and Hedberg, S. E. (1965). Colonic cancer in ulcerative colitis and idiopathic colonic cancer. J. Amer. med. Ass., 191, 815-818.

Weckesser, E. C., and Chinn, A. B. (1953). Carcinoma of the colon complicating chronic ulcerative colitis. Ibid., 152, 905-908.

Weston, S. D., and Feit, H. L. (1966). Osseous metastases from carcinoma of the colon with a review of the literature. Dis. Colon Rect., 9, 61-64. 